\title{
A characterization of regular averaging operators and its consequences
}

\author{
by \\ Spiros A. Argyros and Alexander D. Arvanitakis (Athens)
}

\begin{abstract}
We present a characterization of continuous surjections, between compact metric spaces, admitting a regular averaging operator. Among its consequences, concrete continuous surjections from the Cantor set $\mathcal{C}$ to $[0,1]$ admitting regular averaging operators are exhibited. Moreover we show that the set of this type of continuous surjections from $\mathcal{C}$ to $[0,1]$ is dense in the supremum norm in the set of all continuous surjections. The nonmetrizable case is also investigated. As a consequence, we obtain a new characterization of Eberlein compact sets.
\end{abstract}

1. Introduction. One of the most important results in the isomorphic theory of Banach spaces, due to A. Milyutin, asserts that if $K_{1}, K_{2}$ are uncountable compact metric spaces then the spaces $C\left(K_{1}\right), C\left(K_{2}\right)$ are isomorphic. This result became widely known through A. Pełczyński's monograph [16] and the proof presented by Pełczyński requires the following steps:

Denoting by $\mathcal{C}$ the Cantor set $\{0,1\}^{\mathbb{N}}$ it is enough to show that every $C(K)$ with $K$ an uncountable compact metric space is isomorphic to $C(\mathcal{C})$. Pełczyński's decomposition method (see for example [12, Theorem 2.a.3]) reduces this problem to the complemented embedding of $C(K)$ in $C(\mathcal{C})$ and vice versa. To handle these complementation problems, Pełczyński introduced the concepts of regular averaging and regular extension operators ([16, Definitions 1.1, 2.1]) defined as follows:

Definition 1. (1) Let $K, L$ be compact spaces and $\phi: L \rightarrow K$ a continuous onto map. We say that $\phi$ admits a regular averaging operator if there exists a positive linear operator $u: C(L) \rightarrow C(K)$ with $u\left(1_{L}\right)=1_{K}$ and $u \phi^{\circ}=\mathrm{id}_{C(K)}$, where $\phi^{\circ}: C(K) \rightarrow C(L)$ is the operator induced by $\phi$.

(2) If $\phi: L \rightarrow K$ is one-to-one and continuous, we say that $\phi$ admits a regular extension operator if there exists a positive linear operator $u$ : $C(L) \rightarrow C(K)$ with $u\left(1_{L}\right)=1_{K}$ and $\phi^{\circ} u=\mathrm{id}_{C(L)}$.

2000 Mathematics Subject Classification: Primary 46E15, 54C55; Secondary 28B20. 
Milyutin's Lemma is the assertion that there exists a continuous surjection $\phi: \mathcal{C} \rightarrow[0,1]$ that admits a regular averaging operator. From this, it was deduced that for every compact metric $K$, there exists $\phi: \mathcal{C} \rightarrow K$ continuous onto which also admits a regular averaging operator. Hence it was concluded that for every compact metric $K, C(K)$ can be embedded in $C(\mathcal{C})$ as a complemented subspace.

The second complementation follows from Michael's Selection Theorem (see [14]) that asserts that every embedding $\phi: L \rightarrow K$ with $L$ compact metric and $K$ compact admits a regular extension operator.

For general, not necessarily metrizable compact spaces, we recall the following results:

The first, due to A. Pełczyński [16], asserts that if $\phi_{\gamma}: L_{\gamma} \rightarrow K_{\gamma}, \gamma \in \Gamma$, admits a regular averaging (extension) operator, then the same remains valid for the function $\Phi=\prod \phi_{\gamma}: \prod L_{\gamma} \rightarrow \prod K_{\gamma}$.

S. Ditor [4] has shown that for every compact $K$ there exists a totally disconnected compact $L$ with topological weight equal to that of $K$ and a continuous surjection $\phi: L \rightarrow K$ which admits a regular averaging operator. (Here, by the term topological weight, we mean the least cardinal such that there exists a base of $K$ of that cardinality.)

As we show in this paper, Ditor's Theorem is a direct consequence of the theorem of Pełczyński mentioned above. In spite of this, Ditor's proof contains ideas important to us for proving our results.

In what follows, a totally disconnected space $L$ will be called a Ditor space for a compact space $K$ if the topological weight of $L$ equals that of $K$, and moreover there exists a continuous surjection $\phi: L \rightarrow K$ that admits a regular averaging operator. Ditor's Theorem implies that for any compact $K$, there exists at least one Ditor space for $K$.

The third result, due to R. Haydon [9], concerns regular extension operators, and characterizes those compact spaces $L$ such that every embedding of $L$ in another compact space $K$ admits a regular extension operator.

It is worthwhile to mention here that the question whether or not for an arbitrary compact $K$, there exists a totally disconnected compact $L$ such that $C(K) \cong C(L)$, remains open. A discussion concerning this problem is included in the last part of the present paper.

Additional information for the compact case is contained in [5], [9], [10] and $[6]$.

Related results for non-compact topological spaces can be found in [7], [18] and [20]. The interested reader can also consult the survey paper [17].

Returning to the metrizable case, we point out that the complemented embedding of $C(K)$ into $C(\mathcal{C})$ through a regular averaging operator is in part topological, in the sense that the embedding $\phi^{\circ}: C(K) \rightarrow C(\mathcal{C})$ results from the continuous surjection $\phi: \mathcal{C} \rightarrow K$, and in part functional-analytic. 
We mention that Y. Benyamini [3] has a proof of this result using exclusively techniques from Banach space theory.

The third section of the present paper is mainly devoted to a systematic study of continuous surjections between compact metric spaces which admit regular averaging operators.

The first result we should mention is a topological characterization of continuous surjections between compact metric spaces, admitting regular averaging operators. This characterization is as follows:

TheOREM 2. Let $L$ be a compact metric space and $\phi: L \rightarrow K$ continuous onto. Then the following are equivalent:

(1) The map $\phi$ admits a regular averaging operator.

(2) There exists a basis $\mathcal{U}$ of $L$, containing $L$ and consisting of open sets, and a family $\left\{G_{U}: U \in \mathcal{U}\right\}$ of open subsets of $K$, such that:

(a) $G_{L}=K$.

(b) For each $U \in \mathcal{U}, G_{U} \subset \phi(U)$.

(c) Whenever $U, U_{1}, \ldots, U_{n}$ are members of $\mathcal{U}$ and $U \subset \bigcup_{i=1}^{n} U_{i}$ then also $G_{U} \subset \bigcup_{i=1}^{n} G_{U_{i}}$.

(3) For every basis $\mathcal{U}$ of $L$ containing $L$ and consisting of open sets, there is a family $\left\{G_{U}: U \in \mathcal{U}\right\}$ of open subsets of $K$ satisfying (a)-(c) above.

Condition (2) in the above characterization is useful to establish that some $\phi$ does admit a regular averaging operator, while condition (3) is used to show that $\phi$ does not admit such an operator.

The proof of the above theorem is based on a selection theorem for multivalued maps (Theorem 10) proved in this paper. This selection theorem, probably of independent interest, follows from Lemma 8, the proof of which is inspired by Ditor's ideas in proving his theorem mentioned above.

With Theorem 2 we give an answer to a problem posed by Pełczyński [16]. A different characterization was also obtained by Ditor [5].

Theorem 2 implies that for every $1 / 2<r<1$ the surjection $\phi_{r}: \mathcal{C} \rightarrow$ $[0,1]$ defined as

$$
\phi_{r}\left(\left(\varepsilon_{n}\right)_{n}\right)=(1-r) \sum_{n=1}^{\infty} \varepsilon_{n} r^{n-1}
$$

admits a regular averaging operator and also that for $r=1 / 2$ this is no longer valid.

These functions will be used in the next section to give a precise description of Ditor spaces for certain compact spaces.

A second result included in this section concerns a question posed to us by A. Pełczyński and states the following: 
Theorem 3. The set $\mathcal{R} \mathcal{A}$ of continuous surjections $\psi: \mathcal{C} \rightarrow[0,1]$ that admit regular averaging operators is dense in the supremum norm in the set of all continuous surjections.

As the referee pointed out to us, the above set is residual. This is a consequence of certain general results in Dimension Theory and a more detailed discussion is presented in Remark 16.

In the last part of this paper, we start with a short description of the proof of Pełczyński's Theorem mentioned above and then we show how Ditor's Theorem is obtained as a direct consequence of Pełczyński's Theorem.

Next we prove that under some conditions concerning the compact space $K$, there exists a Ditor space for $K$, homeomorphic to a closed subspace of $K^{\mathbb{N}}$ (Proposition 22). From this we conclude that if $K$ belongs to one of the classes of Eberlein, Gul'ko, Talagrand or Corson compacta, then there exists a Ditor space for $K$ also belonging to the same class. Using this we obtain a new characterization of Eberlein compact sets (recall that a compact space $K$ is called scattered if every closed subset of $K$ has an isolated point):

THEOREM 4. Let $K$ be a compact space. Then $K$ is Eberlein compact if and only if there exists a scattered Eberlein compact $S$ such that $K$ can be embedded in $\mathcal{P}(S)$.

Finally, for the unit ball $B_{p}$ of $\ell_{p}(\Gamma)(1 \leq p<\infty)$ endowed with the pointwise topology, we give a concrete description of a Ditor space which in the case $p=1$ takes the form

$L=\left\{x \in \ell_{1}(\Gamma \times \mathbb{N}):\|x\| \leq 1\right.$ and $\left.\forall(\gamma, n) \in \Gamma \times \mathbb{N}, x(\gamma, n) \in\left\{-r_{n}, 0, r_{n}\right\}\right\}$, where $\left(r_{n}\right)_{n}$ are fixed positive numbers summing up to 1 . Clearly $L$ is embeddable in $B_{1}$.

2. Notation and definitions. Let $L, K$ be Hausdorff compact spaces and $\phi: L \rightarrow K$ a continuous map. We denote by $\phi^{\circ}: C(K) \rightarrow C(L)$ the map $\phi^{\circ}(f)=f \phi$. It is well known that $\phi^{\circ}$ is a bounded linear operator and moreover if $\phi$ is onto then $\phi^{\circ}$ is an isometric embedding; if $\phi$ is one-to-one then $\phi^{\circ}$ is onto.

A bounded operator $u: C(L) \rightarrow C(K)$ is called regular provided $u(f) \geq 0$ whenever $f \geq 0$ and $u\left(1_{L}\right)=1_{K}$, where $1_{L}: L \ni l \mapsto 1 \in \mathbb{R}$ is the constantly 1 function on $L$.

We say that $\phi$ admits a regular averaging operator if there exists a regular operator $u: C(L) \rightarrow C(K)$ such that $u \phi^{\circ}=\operatorname{id}_{C(K)}$ (where $\operatorname{id}_{X}$ denotes the identity map on $X)$. Also $\phi$ admits a regular extension operator if there exists a regular operator $u: C(L) \rightarrow C(K)$ such that $\phi^{\circ} u=\mathrm{id}_{C(L)}$.

We say that $\phi$ admits a choice function if $\phi$ is onto and there exists a continuous map $s: K \rightarrow L$ such that $s(k) \in \phi^{-1}(k)$ or equivalently $\phi s=\operatorname{id}_{K}$. 
We denote by $\mathcal{M}(K)$ the regular measures on $K$. It is well known by Riesz's Representation Theorem that $\mathcal{M}(K)$ can be identified with $C(K)^{*}$, the dual of $C(K)$. Also, $\mathcal{P}(K)$ denotes the regular probability measures on $K$. Unless otherwise stated $\mathcal{P}(K)$ will be endowed with the weak-* topology.

We denote by $\delta_{k}$ the Dirac measure at $k \in K$, and $\phi^{*}$ stands for the restriction $\left(\phi^{\circ}\right)^{*} \mid \mathcal{P}(L): \mathcal{P}(L) \rightarrow \mathcal{P}(K)$ of the dual map of $\phi^{\circ}$ to $\mathcal{P}(L)$.

3. The metrizable case. Let us begin with some observations regarding the function $\phi^{*}$ introduced in the previous section. Recall that for a continuous map $\phi: L \rightarrow K$ we have denoted by $\phi^{*}: \mathcal{P}(L) \rightarrow \mathcal{P}(K)$ the induced affine map. Actually $\phi^{*}$ could also be defined as $\phi^{*}(p)(A)=p\left(\phi^{-1}(A)\right)$, for every $p \in \mathcal{P}(L)$ and $A \subset K$ a Borel measurable set. Hence we easily see that if $p \in \mathcal{P}(L)$, then $\operatorname{supp} \phi^{*}(p) \subset \phi(\operatorname{supp} p)$. These observations lead to the proof of the next lemma which will be repeatedly used.

Lemma 5. Let $\phi: L \rightarrow K$ be a continuous map with $L$ and $K$ Hausdorff compact spaces. Assume that $M$ is a closed subset of $K$. Then $\left(\phi^{*}\right)^{-1}(\mathcal{P}(M))$ $=\mathcal{P}\left(\phi^{-1}(M)\right)$.

In the next proposition we give some useful equivalent statements for regular averaging and regular extension operators which are well known (see [9]). For completeness we indicate briefly how we can obtain them.

Proposition 6. Let $L, K$ be Hausdorff compact spaces and $\phi: L \rightarrow K$ a continuous map.

(1) Assume that $\phi$ is onto. Then the following are equivalent:

(a) $\phi$ admits a regular averaging operator.

(b) $\phi^{*}$ admits a choice function, i.e. there exists a one-to-one continuous map $s: \mathcal{P}(K) \rightarrow \mathcal{P}(L)$ such that $\phi^{*} s=\operatorname{id}_{\mathcal{P}(K)}$.

(c) There exists a continuous map $v: K \rightarrow \mathcal{P}(L)$ such that $\phi^{*} v(k)=$ $\delta_{k}$ for all $k \in K$.

(d) There exists a continuous map $u: K \rightarrow \mathcal{P}(L)$ such that $u(k)$ is supported by $\phi^{-1}(k)$.

(2) Assume that $\phi$ is one-to-one. Then the following are equivalent:

(a) $\phi$ admits a regular extension operator.

(b) $\phi^{*}$ is a choice function for some continuous $s: \mathcal{P}(K) \rightarrow \mathcal{P}(L)$, i.e. $s \phi^{*}=\mathrm{id}_{\mathcal{P}(L)}$.

(c) There exists a continuous map $v: K \rightarrow \mathcal{P}(L)$ such that $v \phi(l)=\delta_{l}$ for all $l \in L$.

Proof. (1) Lemma 5 shows that condition (1)(c) is equivalent to (1)(d). (1) (a) $\Rightarrow(1)(\mathrm{b})$. Let $u$ be a regular averaging operator for $\phi$. Then $u \phi^{\circ}=$ $\operatorname{id}_{C(K)}$ and consequently $\left(\phi^{\circ}\right)^{*} u^{*}=\operatorname{id}_{\mathcal{M}(K)}$. Therefore it suffices to show 
that for all $p \in \mathcal{P}(K), u^{*}(p) \in \mathcal{P}(L)$, since then the map $s=u^{*} \mid \mathcal{P}(K)$ would be the choice function for $\phi^{*}$. Observe that for $f \in C(L), f \geq 0$, we have $u^{*}(p)(f)=p(u(f)) \geq 0$ and hence $u^{*}(p)$ is a positive measure. Moreover $u^{*}(p)\left(1_{L}\right)=p\left(u\left(1_{L}\right)\right)=p\left(1_{K}\right)=1$ and therefore $u^{*}(p)$ is a probability measure.

$(1)(\mathrm{b}) \Rightarrow(1)(\mathrm{c})$. Let $s$ be a choice function for $\phi^{*}$. For $j: K \ni k \mapsto \delta_{k} \in$ $\mathcal{P}(K)$, it is easy to see that $v=s j$ is the required map.

$(1)(\mathrm{c}) \Rightarrow(1)(\mathrm{a})$. Define $u: C(L) \rightarrow C(K)$ by $u(f)(k)=v(k)(f)$. It is easy to see that $u(f)$ is indeed a continuous function on $K$ and that $u$ is a bounded linear operator such that $u(f) \geq 0$ whenever $f \geq 0$ and $u\left(1_{L}\right)=1_{K}$. Moreover for $f \in C(K), u \phi^{\circ}(f)(k)=v(k)\left(\phi^{\circ}(f)\right)=\phi^{*} v(k)(f)=\delta_{k}(f)=$ $f(k)$ for all $k \in K$, so that $u \phi^{\circ}(f)=f$ and hence $u \phi^{\circ}=\operatorname{id}_{C(K)}$.

(2) $(2)(\mathrm{a}) \Rightarrow(2)(\mathrm{b})$. Let $u$ be a regular extension operator for $\phi$. Then $\phi^{\circ} u=\operatorname{id}_{C(L)}$ and consequently $u^{*}\left(\phi^{\circ}\right)^{*}=\operatorname{id}_{\mathcal{M}(L)}$. As before, if $p \in \mathcal{P}(K)$, then $u^{*}(p) \in \mathcal{P}(L)$ and hence $s=u^{*} \mid \mathcal{P}(K)$ is the required map.

$(2)(\mathrm{b}) \Rightarrow(2)(\mathrm{c})$. Let $s: \mathcal{P}(K) \rightarrow \mathcal{P}(L)$ be such that $s \phi^{*}=\mathrm{id}_{\mathcal{P}(L)}$. For $j: K \ni k \mapsto \delta_{k} \in \mathcal{P}(K)$ simply set $v=s j$.

$(2)(\mathrm{c}) \Rightarrow(2)(\mathrm{a})$. Define $u: C(L) \rightarrow C(K)$ by $u(f)(k)=v(k)(f)$. Then $u$ is a regular operator and for $f \in C(L)$ and $l \in L, \phi^{\circ} u(f)(l)=u(f)(\phi(l))=$ $v \phi(l)(f)=\delta_{l}(f)=f(l)$, hence $\phi^{\circ} u=\operatorname{id}_{C(L)}$.

Next we recall the definition of a tree and some relevant notation. A tree of height $\omega$ is a set partially ordered by a relation $\prec$ such that for every $t \in T$, the set $\{s \in T: s \prec t\}$ is linearly ordered and finite.

If $t \in T$, then the set of immediate successors of $t$ will be denoted by $S_{t}$. We say that $T$ is finitely branching if $S_{t}$ is finite for every $t \in T$. We denote by $\mathcal{B}(T)$ the set of branches of $T$, namely of all maximal linearly ordered subsets of $T ; \mathcal{B}(T)$ is naturally topologized by the sets $V_{t}=\{b \in \mathcal{B}(T): t \in b\}$.

It can be easily shown that $T$ is finitely branching if and only if $\mathcal{B}(T)$ is compact. In what follows we assume that every $T$ considered has a unique minimal element, denoted by $r(T)$.

Finally for $t \in T$, we denote by $|t|$ the cardinality of the set $\{s \in T$ : $s \prec t$ and $s \neq t\}$ and for $b \in \mathcal{B}(T)$ (respectively $t \in T$ ) we denote by $b \mid n$ (resp. $t \mid n$ ) the unique $s \in b$ (resp. $s \prec t$ ) such that $|s|=n$.

The following lemma is well known:

Lemma 7. Assume that $T$ is a finitely branching tree of height $\omega$ and to every $t \in T$ there corresponds a non-negative number $\lambda_{t}$ such that:

(1) $\lambda_{r(T)}=1$,

(2) $\sum\left\{\lambda_{s}: s \in S_{t}\right\}=\lambda_{t}$ for every $t \in T$.

Then there exists a unique regular Borel probability measure $p$ on $\mathcal{B}(T)$ such that for every $t \in T, p\left(V_{t}\right)=\lambda_{t}$ (where as above $V_{t}=\{b \in \mathcal{B}(T): t \in b\}$ ). 
For a subset $F$ of a topological space $X$, we denote by $\operatorname{int}(F)$ the interior of $F$. If $F_{2} \subset F_{1} \subset X$ then $\operatorname{int}_{F_{1}}\left(F_{2}\right)$ denotes the relative interior of $F_{2}$ in $F_{1}$.

Also, for a map $f$ defined on a topological space $K$, we denote by $\operatorname{supp}(f)$ the closure in $K$ of the set $\{k \in K: f(k) \neq 0\}$.

Lemma 8. Let $T$ be as in Lemma 7 and $K$ a normal topological space. Assume that to each $t \in T$ an open subset $U_{t}$ of $K$ has been assigned, such that

(1) $U_{r(T)}=K$.

(2) For every $t \in T, U_{t}=\bigcup\left\{U_{s}: s \in S_{t}\right\}$.

For a fixed $k \in K$, set $T(k)=\left\{t \in T: k \in U_{t}\right\}$. Then there exists a continuous function $p: K \rightarrow\left(\mathcal{P}(\mathcal{B}(T)), w^{*}\right)$ such that for every $k \in K, p(k)$ is supported by $\mathcal{B}(T(k))$.

Proof. We define, inductively on $|t|$, a closed subset $F_{t}$ of $U_{t}$ such that for any $t \in T$,

$$
F_{t}=\bigcup\left\{\operatorname{int}_{F_{t}}\left(F_{s}\right): s \in S_{t}\right\} .
$$

We set $F_{r(T)}=U_{r(T)}=K$. Assuming that $F_{t}$ has been defined for any $t$ in the $n$th level of $T$, observe that $\left\{U_{s} \cap F_{t}: s \in S_{t}\right\}$ is a relatively open covering of $F_{t}$. Since $F_{t}$ is itself a normal space, we can find a closed $F_{s} \subset U_{s} \cap F_{t}$ such that $\left\{\operatorname{int}_{F_{t}}\left(F_{s}\right): s \in S_{t}\right\}$ is also an open covering of $F_{t}$.

Since $S_{t}$ is a finite set and $F_{t}$ is normal, there exists a partition of unity $\left\{f_{s}: s \in S_{t}\right\}$ defined on $F_{t}$ and subordinate to the covering $\left\{\operatorname{int}_{F_{t}}\left(F_{s}\right)\right.$ : $\left.s \in S_{t}\right\}$. Every $f_{s}$ is a continuous function on $F_{t}$ and we extend it to $K$ (not necessarily continuously) by setting it constantly zero outside $F_{t}$. We shall denote this extension also by $f_{s}$. Also, for $k \in K$ and $t \in T$ we set

$$
\lambda_{t}(k)=\prod_{s \preceq t} f_{s}(k) .
$$

So, for a fixed $t \in T$ a real-valued map $K \ni k \mapsto \lambda_{t}(k)$ is defined. We assert that for $t \in T$, the function $\lambda_{t}$ is continuous on $K$. To see this, we prove inductively on $|t|$ that $\operatorname{supp}\left(\lambda_{t}\right) \subset \operatorname{int}_{K}\left(F_{t}\right)$. This immediately implies the continuity of $\lambda_{t}$, since $K=\left(K \backslash \operatorname{supp}\left(\lambda_{t}\right)\right) \cup \operatorname{int}_{K}\left(F_{t}\right)$ and $\lambda_{t}$ is by definition continuous on both open sets.

The assertion is clear for $|t| \leq 1$ by the definition of $f_{t}$. The inductive step goes as follows: For $t \in S_{s}$,

$$
\left\{k \in K: \lambda_{t}(k)>0\right\}=\left\{k \in K: \lambda_{s}(k)>0\right\} \cap\left\{k \in K: f_{t}(k)>0\right\}
$$

and thus

$$
\operatorname{supp} \lambda_{t} \subset \operatorname{supp} \lambda_{s} \cap \operatorname{supp} f_{t} \subset \operatorname{int}_{K}\left(F_{s}\right) \cap \operatorname{int}_{F_{s}}\left(F_{t}\right) \subset \operatorname{int}_{K}\left(F_{t}\right) .
$$

Observe also that for each $k \in K$ and $t \in T, \sum\left\{\lambda_{s}(k): s \in S_{t}\right\}=\lambda_{t}(k)$. Let 
$p$ be the map

$$
p: K \ni k \mapsto p(k) \in\left(\mathcal{P}(\mathcal{B}(T)), w^{*}\right)
$$

such that for every $t, p(k)\left(V_{t}\right)=\lambda_{t}(k)$. Its existence is assured by Lemma 7 . Moreover this map is continuous. This follows from the continuity of the maps $k \mapsto \lambda_{t}(k)$, since the set $\left\{\chi_{V_{t}}: t \in T\right\}$ of characteristic functions spans a dense subset of $C(\mathcal{B}(T))$ and $p(k)\left(\chi_{V_{t}}\right)=p(k)\left(V_{t}\right)=\lambda_{t}(k)$ by Lemma 7 .

Next, fixing $n \in \mathbb{N}$ and $k \in K$, we set

$$
G_{n}(k)=\bigcup\left\{V_{t}: t \in T(k) \text { and }|t|=n\right\} .
$$

If $k \notin U_{t}$ for some $t$, then $p(k)\left(V_{t}\right)=\lambda_{t}(k)=0$, since supp $\lambda_{t} \subset F_{t} \subset U_{t}$. It follows that for every $n \in \mathbb{N}, p(k)$ is supported by $G_{n}(k)$. Since moreover $\left\{G_{1}(k), G_{2}(k), \ldots\right\}$ is a decreasing sequence of open subsets of $\mathcal{B}(T)$ and $\bigcap_{n} G_{n}(k)=\mathcal{B}(T(k))$, we have

$$
p(k)(\mathcal{B}(T(k)))=\lim _{n} p(k)\left(G_{n}(k)\right)=1
$$

and this concludes the proof.

For a set-valued map $\Phi: K \rightarrow 2^{L}$ and $M \subset L$, we denote by $\Phi^{-1}(M)$ the set $\{k \in K: \Phi(k) \cap M \neq \emptyset\}$.

Theorem 9. Let $T$ be as in Lemma 7 and $K$ a normal topological space. Let moreover $\Phi: K \rightarrow 2^{\mathcal{B}(T)}$ be a set-valued map such that for each $k \in K$, $\Phi(k)$ is closed. Then the following are equivalent:

(1) There exists a continuous map $p: K \rightarrow\left(\mathcal{P}(\mathcal{B}(T)), w^{*}\right)$ such that for each $k \in K, p(k)$ is supported by $\Phi(k)$.

(2) For each $t \in T$, there exists an open subset $G_{t}$ of $\Phi^{-1}\left(V_{t}\right)$ such that:

(a) $G_{r(T)}=K$.

(b) For any $t \in T, G_{t}=\bigcup\left\{G_{s}: s \in S_{t}\right\}$.

(We recall that $V_{t}$ denotes the set $\{b \in \mathcal{B}(T): t \in b\}$.)

Proof. For the implication $(2) \Rightarrow(1)$, the previous lemma ensures the existence of a continuous $p: K \rightarrow\left(\mathcal{P}(\mathcal{B}(T)), w^{*}\right)$ such that for each $k \in K$, $p(k)$ is supported by $\mathcal{B}(T(k))$. In this case

$$
T(k)=\left\{t \in T: k \in G_{t}\right\} \subset\left\{t \in T: \Phi(k) \cap V_{t} \neq \emptyset\right\}
$$

and since $\Phi(k)$ is closed, $\mathcal{B}(T(k)) \subset \Phi(k)$. Hence $p(k)$ is also supported by $\Phi(k)$ and this proves (1).

For the converse implication, let $p$ be as in (1) and set

$$
G_{t}=p^{-1}\left(\left\{\mu \in \mathcal{P}(\mathcal{B}(T)): \mu\left(V_{t}\right)>0\right\}\right) .
$$

Since $p$ is continuous, $G_{t}$ is an open subset of $K$, and clearly $G_{r(T)}=K$. Since moreover $V_{t}=\bigcup\left\{V_{s}: s \in S_{t}\right\}$, also $G_{t}=\bigcup\left\{G_{s}: s \in S_{t}\right\}$. Observe also that for $k \in G_{t}, p(k)\left(V_{t}\right)>0$ and by assumption $p(k)$ is supported by $\Phi(k)$. Hence $\Phi(k) \cap V_{t} \neq \emptyset$ and therefore $k \in \Phi^{-1}\left(V_{t}\right)$. 
We shall make use of the previous theorem to prove the more general:

TheORem 10. Let $K$ be a normal topological space and $L$ a compact metrizable space. Let moreover $\Phi: K \rightarrow 2^{L}$ be a set-valued map such that for each $k \in K, \Phi(k)$ is closed. Then the following are equivalent:

(1) There exists a continuous map $p: K \rightarrow\left(\mathcal{P}(L), w^{*}\right)$ such that for each $k \in K, p(k)$ is supported by $\Phi(k)$.

(2) There exists a basis $\mathcal{U}$ of $L$, containing $L$ and consisting of open sets, and a family $\left\{G_{U}: U \in \mathcal{U}\right\}$ of open subsets of $K$ such that:

(a) $G_{L}=K$.

(b) For each $U \in \mathcal{U}, G_{U} \subset \Phi^{-1}(U)$.

(c) Whenever $U, U_{1}, \ldots, U_{n}$ are members of $\mathcal{U}$ and $U \subset \bigcup_{i=1}^{n} U_{i}$, then also $G_{U} \subset \bigcup_{i=1}^{n} G_{U_{i}}$.

(3) For every basis $\mathcal{U}$ of $L$ containing $L$ and consisting of open sets, there exists a family $\left\{G_{U}: U \in \mathcal{U}\right\}$ of open subsets of $K$ such that (a)-(c) above are fulfilled.

Proof. For the implication $(1) \Rightarrow(3)$, for $U \in \mathcal{U}$, set

$$
G_{U}=p^{-1}(\{\mu \in \mathcal{P}(L): \mu(U)>0\}) .
$$

Since $p$ is continuous, $G_{U}$ is open and (a) and (c) follow immediately.

To prove (b), for $k \in G_{U}, p(k)(U)>0$ and $p(k)$ is supported by $\Phi(k)$. Thus $\Phi(k) \cap U \neq \emptyset$ and hence $k \in \Phi^{-1}(U)$.

The implication $(3) \Rightarrow(2)$ is trivial.

$(2) \Rightarrow(1)$. We inductively define a tree $T$ of elements of $\mathcal{U}$ as follows: We set $r(T)=L$. Assuming that the $n$th level $T_{n}$ of $T$ has been defined, for $U \in T_{n}$ we choose $U_{1}, \ldots, U_{k}$ in $\mathcal{U}$ such that

$$
\operatorname{diam}\left(\bar{U}_{i}\right)<\frac{1}{2^{n+1}}, \quad \bar{U} \subset \bigcup_{i=1}^{k} U_{i}, \quad U \cap U_{i} \neq \emptyset .
$$

Then $U_{1}, \ldots, U_{k}$ are exactly the immediate successors of $U$.

It is a direct consequence of the definition and the compactness of $L$ that for any $b \in \mathcal{B}(T)$ the set $\lim \{\bar{U}: U \in b\}$ in the Hausdorff metric is a singleton. We define a map $\phi: \mathcal{B}(T) \rightarrow L$ by

$$
\phi: \mathcal{B}(T) \ni b \mapsto \phi(b) \in \lim \{\bar{U}: U \in b\} .
$$

It can be easily checked that $\phi$ is continuous and onto.

Next we define $\Psi: K \rightarrow 2^{\mathcal{B}(T)}$ by $\Psi(k)=\phi^{-1}(\Phi(k))$. Clearly, $\Psi$ is a set-valued map and for every $k \in K, \Psi(k)$ is closed.

Also, for $U \in T$, we set

$$
R_{U}=\bigcap\left\{G_{U^{\prime}}: U^{\prime} \preceq U\right\}
$$


(where $\prec$ is the partial order of the tree $T$ ). Setting also $V_{U}=\{b \in \mathcal{B}(T)$ : $U \in b\}$, we observe that condition (2) of the inductive definition of the tree implies that $\phi\left(V_{U}\right) \supset \bar{U}$. Next we check that the family $\left\{R_{U}: U \in T\right\}$ satisfies the conditions of the previous theorem. First we have $\Psi^{-1}\left(V_{U}\right)=$ $\Phi^{-1}(\bar{U})$. Indeed,

$$
\begin{aligned}
\Psi^{-1}\left(V_{U}\right) & =\left\{k \in K: \Psi(k) \cap V_{U} \neq \emptyset\right\}=\left\{k \in K: \phi^{-1}(\Phi(k)) \cap V_{U} \neq \emptyset\right\} \\
& =\left\{k \in K: \Phi(k) \cap \phi\left(V_{U}\right) \neq \emptyset\right\} \supset\{k \in K: \Phi(k) \cap \bar{U} \neq \emptyset\}=\Phi^{-1}(\bar{U}) .
\end{aligned}
$$

Since $G_{U} \subset \Phi^{-1}(U)$, by our hypothesis, it follows that also $R_{U} \subset \Psi^{-1}\left(V_{U}\right)$. Moreover if $U_{1}, \ldots, U_{n}$ are the immediate successors of $U$, then by the definition of the tree $T, U \subset \bigcup_{i=1}^{n} U_{i}$, hence $G_{U} \subset \bigcup_{i=1}^{n} G_{U_{i}}$ and thus

$$
\begin{aligned}
R_{U} & =\bigcap\left\{G_{U^{\prime}}: U^{\prime} \preceq U\right\}=\bigcap\left\{G_{U^{\prime}}: U^{\prime} \prec U\right\} \cap G_{U} \\
& =\bigcap\left\{G_{U^{\prime}}: U^{\prime} \prec U\right\} \cap \bigcup_{i=1}^{n}\left(G_{U} \cap G_{U_{i}}\right) \\
& =\bigcup_{i=1}^{n}\left\{G_{U^{\prime}}: U^{\prime} \preceq U_{i}\right\}=\bigcup_{i=1}^{n} R_{U_{i}} .
\end{aligned}
$$

Note moreover that $R_{r(T)}=R_{L}=G_{L}=K$ by our hypothesis.

Thus the previous theorem can be applied to give a continuous map $p^{\prime}: K \rightarrow\left(\mathcal{P}(\mathcal{B}(T)), w^{*}\right)$ such that $p^{\prime}(k)$ is supported by $\Psi(k)$. By Lemma 5 ,

$$
\mathcal{P}(\Psi(k))=\mathcal{P}\left(\phi^{-1}(\Phi(k))\right)=\left(\phi^{*}\right)^{-1}(\mathcal{P}(\Phi(k)))
$$

and hence $\phi^{*} p^{\prime}(k)$ is a probability measure supported by $\Phi(k)$ as required. Thus $p=\phi^{*} p^{\prime}$ is the appropriate map.

From the above theorem, we obtain the characterization of continuous surjections admitting regular averaging operators, mentioned in the introduction:

Proof of Theorem 2. For $k \in K$ we set $\Phi(k)=\phi^{-1}(k)$. In this case $\Phi^{-1}(U)=\phi(U)$. Thus the existence of a continuous map $p: K \rightarrow\left(\mathcal{P}(L), w^{*}\right)$ such that $p(k)$ is supported by $\Phi(k)=\phi^{-1}(k)$ is equivalent by Proposition 6 $(1)(\mathrm{a}) \Leftrightarrow(1)(\mathrm{d})$ to the existence of a regular averaging operator for $\phi$.

Theorem 11. Let $1 / 2<r<1$ and $r_{n}=(1-r) r^{n-1}, n \in \mathbb{N}$. Then the map

$$
\phi:\{0,1\} \ni x \mapsto \sum_{n=1}^{\infty} x(n) r_{n} \in[0,1]
$$

admits a regular averaging operator. 
Proof. For $t$ an element of the dyadic tree $\mathcal{D}$ (i.e. a finite sequence of 0 's and 1's), let

$$
V_{t}=\left\{b \in\{0,1\}^{\mathbb{N}}: b(1)=t(1), \ldots, b(|t|)=t(|t|)\right\}
$$

be the basic clopen subsets of the Cantor space. Then

$$
\phi\left(V_{t}\right)=\left[\sum_{i=1}^{|t|} r_{i} t(i), \sum_{i=1}^{|t|} r_{i} t(i)+\sum_{i=|t|+1}^{\infty} r_{i}\right]=\left[\sum_{i=1}^{|t|} r_{i} t(i), \sum_{i=1}^{|t|} r_{i} t(i)+r^{|t|}\right] .
$$

Thus denoting by $t \frown i$ the element of $\mathcal{D}$ extending $t$ by $i$, we have

$$
\begin{aligned}
& \phi\left(V_{t-0}\right)=\left[\sum_{i=1}^{|t|} r_{i} t(i), \sum_{i=1}^{|t|} r_{i} t(i)+r^{|t|+1}\right], \\
& \phi\left(V_{t-1}\right)=\left[\sum_{i=1}^{|t|} r_{i} t(i)+(1-r) r^{|t|}, \sum_{i=1}^{|t|} r_{i} t(i)+\sum_{i=|t|+1}^{\infty} r_{i}\right] .
\end{aligned}
$$

Thus setting

$$
G_{t}=\operatorname{int}_{[0,1]}\left(\phi\left(V_{t}\right)\right)=\left(\sum_{i=1}^{|t|} r_{i} t(i), \sum_{i=1}^{|t|} r_{i} t(i)+\sum_{i=|t|+1}^{\infty} r_{i}\right)
$$

we see that $G_{t}=G_{t \frown 0} \cup G_{t-1}$, since $(1-r) r^{|t|}<r^{|t|+1}$. Hence it follows easily from Theorem 2 that $\phi$ admits a regular averaging operator.

REMARK 12. For later use, observe that the map $\phi:\{-1,0,1\}^{\mathbb{N}} \rightarrow$ $[-1,1]$ defined by

$$
\phi(x)= \begin{cases}\sum_{n \in \mathbb{N}}|x(n)| r_{n} & \text { if } x(\min \{n \in \mathbb{N}: x(n) \neq 0\})=1, \\ -\sum_{n \in \mathbb{N}}|x(n)| r_{n} & \text { if } x(\min \{n \in \mathbb{N}: x(n) \neq 0\})=-1, \\ 0 & \text { else, }\end{cases}
$$

where $r_{n}=(1-r) r^{n-1}$, admits a regular averaging operator. This can be proved in a similar manner to the proof of the above theorem.

REMARK 13. On the contrary, for $r=1 / 2$, the dyadic representation

$$
\phi:\{0,1\} \ni x \mapsto \frac{1}{2} \sum_{n=1}^{\infty}\left(\frac{1}{2}\right)^{n-1}
$$

does not admit a regular averaging operator:

Setting $V_{(i)}=\left\{x \in\{0,1\}^{\mathbb{N}}: x(1)=i\right\}$ for $i=0,1$, it is easy to calculate that $\phi\left(V_{(0)}\right)=[0,1 / 2]$ and $\phi\left(V_{(1)}\right)=[1 / 2,1]$. Thus for any open $G_{(0)} \subset \phi\left(V_{(0)}\right)$ and $G_{(1)} \subset \phi\left(V_{(1)}\right)$ we see that $[0,1] \neq G_{(0)} \cup G_{(1)}$. Hence by Theorem 2, condition (3), we conclude that $\phi$ does not admit a regular averaging operator. 
The next theorem is well known and it follows from Michael's Selection Theorem (see [14]). It is used to verify that every homeomorphic embedding of a compact metric space admits a regular extension operator. A unified approach to regular averaging and regular extension operators for general paracompact spaces is presented in [2].

THEOREM 14. Assume that $K$ is a Hausdorff compact space and $M \subset K$ a closed metrizable subset. Then $\mathcal{P}(M)$ is a retract of $\mathcal{P}(K)$.

We conclude this section by proving Theorem 3 .

Proof of Theorem 3. Let $\phi: \mathcal{C} \rightarrow[0,1]$ be continuous onto and $\varepsilon>0$. Consider a finite family $I_{1}, \ldots, I_{n}$ of closed subintervals of $[0,1]$ such that for each $i, \operatorname{diam}\left(I_{i}\right)<\varepsilon$ and moreover

$$
\bigcup_{i=1}^{n} \operatorname{int}_{[0,1]}\left(I_{i}\right)=[0,1] \text { and for every } i_{0}, \quad I_{i_{0}} \backslash \bigcup_{i=1, i \neq i_{0}}^{n} I_{i} \neq \emptyset \text {. }
$$

The rough idea of the proof is to find a partition of $\mathcal{C}$ into clopen non-empty sets $V_{i}$ such that $\phi\left(V_{i}\right) \subset I_{i}$. Since then each $V_{i}$ is homeomorphic to $\mathcal{C}$ and each $I_{i}$ is homeomorphic to $[0,1]$, there exists a continuous onto map $\psi_{i}$ : $V_{i} \rightarrow I_{i}$ which admits a regular averaging operator. We can define $\psi: \mathcal{C} \rightarrow$ $[0,1]$ by $\psi(x)=\psi_{i}(x)$ if $x \in V_{i}$. Using the fact that $\bigcup_{i=1}^{n} \operatorname{int}_{[0,1]}\left(I_{i}\right)=[0,1]$ and moreover that each $\psi_{i}$ admits a regular averaging operator, we can prove that $\psi$ also admits a regular averaging operator. Furthermore, applying the fact that $\operatorname{diam}\left(I_{i}\right)<\varepsilon$, we prove that

$$
\|\psi-\phi\|=\sup \{|\psi(x)-\phi(x)|: x \in \mathcal{C}\}<\varepsilon .
$$

Here is how we accomplish these steps: For notational convenience, set $U_{i}=\operatorname{int}_{[0,1]}\left(I_{i}\right)$. We will define by induction on $i_{0}$ clopen subsets $V_{i}, i \leq i_{0}$, of $\mathcal{C}$ such that $V_{i}$ are pairwise disjoint, and moreover

$$
\bigcup_{i=1}^{i_{0}} V_{i} \cup \bigcup_{i=i_{0}+1}^{n} \phi^{-1}\left(U_{i}\right)=\mathcal{C} \quad \text { and } \quad V_{i} \subset \phi^{-1}\left(U_{i}\right), i \leq i_{0} .
$$

To start with, notice that $\bigcup_{i} \phi^{-1}\left(U_{i}\right)$ covers $\mathcal{C}$ since $\phi$ is onto, so that $\mathcal{C} \backslash$ $\bigcup_{i=2}^{n} \phi^{-1}\left(U_{i}\right)$ is a closed subset of $\phi^{-1}\left(U_{1}\right)$. By standard arguments we can find a clopen $V_{1}$ such that

$$
\mathcal{C} \backslash \bigcup_{i=2}^{n} \phi^{-1}\left(U_{i}\right) \subset V_{1} \subset \phi^{-1}\left(U_{1}\right)
$$

Notice that $V_{1}$ is non-empty since it contains at least $\phi^{-1}\left([0,1] \backslash \bigcup_{i=2}^{n} U_{i}\right)$.

For the induction step, since $\bigcup_{i=1}^{i_{0}-1} V_{i} \cup \bigcup_{i=i_{0}}^{n} \phi^{-1}\left(U_{i}\right)=\mathcal{C}$, it follows that $\mathcal{C} \backslash\left(\bigcup_{i=1}^{i_{0}-1} V_{i} \cup \bigcup_{i=i_{0}+1}^{n} \phi^{-1}\left(U_{i}\right)\right)$ is a non-empty closed subset of $\phi^{-1}\left(U_{i_{0}}\right)$. 
Thus there exists a clopen $V_{i_{0}}$ such that

$$
\mathcal{C} \backslash\left(\bigcup_{i=1}^{i_{0}-1} V_{i} \cup \bigcup_{i=i_{0}+1}^{n} \phi^{-1}\left(U_{i}\right)\right) \subset V_{i_{0}} \subset \phi^{-1}\left(U_{i_{0}}\right)
$$

and clearly in this case $\bigcup_{i=1}^{i_{0}} V_{i} \cup \bigcup_{i=i_{0}+1}^{n} \phi^{-1}\left(U_{i}\right)=\mathcal{C}$.

At the end of course $V_{n}=\mathcal{C} \backslash \bigcup_{i=1}^{n-1} V_{i}$, so that $\left\{V_{i}\right\}_{i}$ is a partition of $\mathcal{C}$ into clopen sets as required, and $\phi\left(V_{i}\right) \subset U_{i} \subset I_{i}$.

For each $i$, fix some continuous $\psi_{i}: V_{i} \rightarrow I_{i}$ which admits a regular averaging operator and define $L$ to be the disjoint union of $I_{i}$ 's, $\bigcup_{i=1}^{n} I_{i} \times\{i\}$. The combined map $\psi^{1}: \mathcal{C} \rightarrow L$, defined by $\psi^{1}(x)=\left(\psi_{i}(x), i\right)$ if $x \in V_{i}$, clearly admits a regular averaging operator. Moreover the natural projection $\psi^{2}: L \rightarrow[0,1]$ defined by $\psi^{2}((x, i))=x$ also admits a regular averaging operator. Let us see why: Fix a partition of unity $\left\{f_{i}: i=1, \ldots, n\right\}$ on $[0,1]$ subordinate to the covering $\left\{U_{i}: i=1, \ldots, n\right\}$ and let $u:[0,1] \rightarrow \mathcal{P}(L)$ be defined by $u(x)=\sum_{i=1}^{n} f_{i}(x) \delta_{(x, i)}$ where as usual $\delta_{(x, i)}$ denotes the Dirac measure supported on $\{(x, i)\}$. Since for a fixed $x \in[0,1], f_{i}(x)>0$ implies $x \in U_{i} \subset I_{i}, u$ is well defined, and since moreover for any $i, \psi^{2}((x, i))=x$, $u(x)$ is a probability measure supported by $\left(\psi^{2}\right)^{-1}(x)$. It is also easy to see that $u$ is continuous.

By Proposition 6, $\psi^{2}$ admits a regular averaging operator, as required. Thus also $\psi=\psi^{2} \psi^{1}: \mathcal{C} \rightarrow[0,1]$ admits a regular averaging operator.

Since $\psi \mid V_{i}=\psi_{i}$ and $\psi_{i}\left(V_{i}\right)=I_{i}$, and since also $\phi\left(V_{i}\right) \subset I_{i}$, for a fixed $x \in \mathcal{C}, \psi(x)$ and $\phi(x)$ belong to the same $I_{i}$ for this particular $i$ for which $x \in V_{i}$. Thus $|\psi(x)-\phi(x)|<\varepsilon$ since $\operatorname{diam}\left(I_{i}\right)<\varepsilon$ and hence $\|\phi-\psi\|<\varepsilon$.

The characterization of the continuous surjections that admit a regular averaging operator yields the following

COROLlaRY 15. Let $f: \mathcal{C} \rightarrow[0,1]$ be a surjection that admits a regular averaging operator. Then the set

$$
\left\{x \in[0,1]: f^{-1}(x) \text { is finite }\right\}
$$

is of the first Baire category in $[0,1]$.

Proof. We inductively prove that for every subinterval $I$ of $[0,1]$ and $n \in \mathbb{N}$, there exists a subinterval $I^{\prime}$ of $I$ and pairwise disjoint clopen subsets $V_{t_{1}}, \ldots, V_{t_{n}}$ of $\mathcal{C}$ such that $f\left(V_{t_{i}}\right) \supset I^{\prime}$ for all $1 \leq i \leq n$. The proof of the inductive step makes use of the characterization obtained in Theorem 2.

The above assertion implies that the set

$$
T_{n}=\left\{x \in[0,1]:\left|f^{-1}(x)\right| \geq n\right\}
$$

contains a dense open set, which immediately yields the desired result.

REMARK 16. It was an open question for us if the set $\mathcal{R} \mathcal{A}$ in the statement of Theorem 3 could contain a dense $G_{\delta}$ set. As mentioned in the in- 
troduction, the referee informed us that it is not possible. Indeed, a general result in Dimension Theory shows that the set $\Psi$ of all continuous surjections $f: \mathcal{C} \rightarrow[0,1]$ with the property that there exists a $y \in[0,1]$ such that $\left|f^{-1}(y)\right|>2$ is of the first Baire category in the set of all surjections [11, Section $45, \mathrm{II}]$.

This result in conjunction with the above corollary implies that the set $\mathcal{R} \mathcal{A}$ is residual.

We thank the referee for bringing this result to our attention.

4. Consequences for some classes of compacta. The next theorem is due to A. Pełczyński and shows how averaging operators and extension operators extend to Cartesian products (see [16]).

Theorem 17. Let $\left\{L_{i}\right\}_{i \in I},\left\{K_{i}\right\}_{i \in I}$ be families of Hausdorff compact topological spaces and for each $i \in I, \phi_{i}: L_{i} \rightarrow K_{i}$ be continuous maps. Let

$$
\phi: L=\prod_{i \in I} L_{i} \ni\left(l_{i}\right)_{i \in I} \mapsto\left(\phi_{i}\left(l_{i}\right)\right)_{i \in I} \in \prod_{i \in I} K_{i}=K .
$$

Then:

(1) If each $\phi_{i}$ is onto and admits a regular averaging operator, then also $\phi$ is onto and admits a regular averaging operator.

(2) If each $\phi_{i}$ is one-to-one and admits a regular extension operator, then also $\phi$ is one-to-one and admits a regular extension operator.

Proof. (1) Clearly $\phi$ is onto. Let $u_{i}$ be a regular averaging operator for $\phi_{i}$. Define $v: K \rightarrow \mathcal{P}(L)$ by $v\left(\left(k_{i}\right)_{i \in I}\right)=\bigotimes_{i \in I} u_{i}^{*}\left(\delta_{k_{i}}\right)$ where we denote by $\bigotimes_{i \in I} u_{i}^{*}\left(\delta_{k_{i}}\right)$ the product measure of $u_{i}^{*}\left(\delta_{k_{i}}\right)$.

To check the continuity of $v$, let $k^{a}=\left(k_{i}^{a}\right)_{i \in I} \rightarrow k=\left(k_{i}\right)_{i \in I}$ be a convergent net and let $\pi_{i}$ be the projection of $L=\prod_{i \in I} L_{i}$ onto the $i$ th coordinate. Then from the Stone-Weierstrass Theorem it suffices to show that for $f \in C(L)$ of the form $f=\pi_{i_{1}}^{\circ}\left(f_{1}\right) \ldots \pi_{i_{m}}^{\circ}\left(f_{m}\right), f_{n} \in C\left(L_{i_{n}}\right)$, $v\left(k^{a}\right)(f) \rightarrow v(k)(f)$. Observe that

$$
\begin{aligned}
v\left(k^{a}\right)(f) & =\int \pi_{i_{1}}^{\circ}\left(f_{1}\right) \ldots \pi_{i_{m}}^{\circ}\left(f_{m}\right) d \bigotimes_{i \in I} u_{i}^{*}\left(\delta_{k_{i}^{a}}\right) \\
& =\int f_{1} d u_{i_{1}}^{*}\left(\delta_{k_{i_{1}}^{a}}\right) \ldots \int f_{m} d u_{i_{m}}^{*}\left(\delta_{k_{i_{m}}^{a}}\right) \\
& \rightarrow \int f_{1} d u_{i_{1}}^{*}\left(\delta_{k_{i_{1}}}\right) \ldots \int f_{m} d u_{i_{m}}^{*}\left(\delta_{k_{i_{m}}}\right)=v(k)(f),
\end{aligned}
$$

which proves the desired result.

Let $k=\left(k_{i}\right)_{i \in I}$ be an element of $K$. Since $u_{i}^{*}\left(\delta_{k_{i}}\right) \in\left(\phi_{i}^{*}\right)^{-1}\left(\delta_{k_{i}}\right)$, it follows according to Lemma 5 that $u_{i}^{*}\left(\delta_{k_{i}}\right)\left(\phi_{i}^{-1}\left(k_{i}\right)\right)=1$ and thus

$$
v(k)\left(\phi^{-1}(k)\right)=\bigotimes_{i \in I} u_{i}^{*}\left(\delta_{k_{i}}\right)\left(\prod_{i \in I} \phi_{i}^{-1}\left(k_{i}\right)\right)=1 .
$$


Hence $v(k)$ is supported by the set $\phi^{-1}(k)$ and according to the same lemma $\phi^{*} v(k)=\delta_{k}$. Now by Proposition 6 , the proof is complete.

(2) Obviously $\phi$ is one-to-one in this case. Let $u_{i}$ be a regular extension operator for $\phi_{i}$ and define $v: K \rightarrow \mathcal{P}(L)$ by $v\left(\left(k_{i}\right)_{i \in I}\right)=\bigotimes_{i \in I} u_{i}^{*}\left(\delta_{k_{i}}\right)$ as before. It suffices to check that for $l=\left(l_{i}\right)_{i \in I} \in L, v(\phi(l))=\delta_{l}$. Observe that $u_{i}^{*}\left(\delta_{\phi_{i}\left(l_{i}\right)}\right)=u_{i}^{*} \phi_{i}^{*}\left(\delta_{l_{i}}\right)=\delta_{l_{i}}$ and hence $v(\phi(l))=\bigotimes_{i \in I} u_{i}^{*}\left(\delta_{\phi_{i}\left(l_{i}\right)}\right)=$ $\bigotimes_{i \in I} \delta_{l_{i}}=\delta_{l}$.

Proposition 18. Assume that $L, K$ are Hausdorff compact spaces, and $\phi: L \rightarrow K$ a continuous onto map which admits a regular averaging operator. Moreover assume that $M$ is a closed subset of $K$. Then the map $\phi \mid \phi^{-1}(M): \phi^{-1}(M) \rightarrow M$ admits a regular averaging operator.

Proof. It suffices to show that $\left(\phi \mid \phi^{-1}(M)\right)^{*}$ admits a choice function. Let $u$ be a choice function for $\phi^{*}$. It suffices to show that $u(\mathcal{P}(M)) \subset \mathcal{P}\left(\phi^{-1}(M)\right)$, since then $u \mid \mathcal{P}(M)$ would be a choice function for $\left(\phi \mid \phi^{-1}(M)\right)^{*}$. This follows immediately from Lemma 5 , since $u(\mathcal{P}(M)) \subset\left(\phi^{*}\right)^{-1}(\mathcal{P}(M))$.

We are now able to derive a proof of the next theorem which is due to S. Ditor [4]. Recall that for a topological space $K$, the topological weight of $K$ is defined to be the smallest cardinal $\mathfrak{m}$ such that $K$ has a base of cardinality $\mathfrak{m}$.

THEOREM 19. If $K$ is an infinite Hausdorff compact space of topological weight $\mathfrak{m}$, then there exists a totally disconnected compact space $L$ of topological weight at most $\mathfrak{m}$ and a continuous onto map $\psi: L \rightarrow K$ which admits a regular averaging operator.

Proof. By Milyutin's Lemma [16, 15], or alternatively by Theorem 11, there exists a continuous map $\phi:\{0,1\}^{\mathbb{N}} \rightarrow[0,1]$ that admits a regular averaging operator. Thus by Theorem 17 there exists a continuous map $\psi:\{0,1\}^{\mathbb{N} \times \mathfrak{m}} \rightarrow[0,1]^{\mathfrak{m}}$ which admits a regular averaging operator. Since $K$ in this case can be considered a subspace of $[0,1]^{\mathfrak{m}}$, Proposition 18 asserts that $\psi \mid \psi^{-1}(K): \psi^{-1}(K) \rightarrow K$ admits a regular averaging operator and clearly $\psi^{-1}(K)$ is a totally disconnected compact space having topological weight at most $\mathfrak{m}$.

REMARK 20. If $K$ is a metrizable compact space, then it may be viewed as a subspace of $[0,1]^{\mathbb{N}}$. Using the same arguments as in the above theorem, we deduce that there exists a closed subspace of $\{0,1\}^{\mathbb{N} \times \mathbb{N}}=\{0,1\}^{\mathbb{N}}$ that is mapped onto $K$ by a function that admits a regular averaging operator. Moreover by a well known theorem this closed subspace of $\{0,1\}^{\mathbb{N}}$ is a retract of $\{0,1\}^{\mathbb{N}}$. Thus there exists a continuous onto $\phi:\{0,1\}^{\mathbb{N}} \rightarrow K$ that admits a regular averaging operator. As explained in the introduction, this result, in conjuction with Theorem 14 and Pełczyński's decomposition method, yields a proof of Milyutin's Theorem. 
The next proposition is an immediate consequence of Proposition 18 and Theorems 11 and 17 .

Proposition 21. Let $K$ be a closed subset of $[0,1]^{\Gamma}, r_{n}=(1 / 3)(2 / 3)^{n-1}$, $n \in \mathbb{N}$ and let $\phi:\{0,1\}^{\Gamma \times \mathbb{N}} \rightarrow[0,1]^{\Gamma}$ be defined by $\phi(x)(\gamma)=\sum_{n=1}^{\infty} x(\gamma, n) r_{n}$. Set $L=\phi^{-1}(K)$. Then $\phi \mid L: L \rightarrow K$ is continuous onto and admits a regular averaging operator.

Assume that $\Gamma$ is a set, $A$ a subset of $\Gamma$ and $x \in[0,1]^{\Gamma}$. By $x\lceil A$ we denote the following element of $[0,1]^{\Gamma}$ :

$$
x\left\lceil A= \begin{cases}x(\gamma) & \text { if } \gamma \in A, \\ 0 & \text { if } \gamma \in \Gamma \backslash A .\end{cases}\right.
$$

If $K \subset[0,1]^{\Gamma}$, then by the adequate hull of $K$, we mean the set

$$
\{x \uparrow A: x \in K \text { and } A \subset \Gamma\}
$$

and we denote it by $\operatorname{ad}(K)$.

It is not difficult to see that if $K$ is a compact subset of $[0,1]^{\Gamma}$, then $\operatorname{ad}(K)$ is also compact.

Using this notation we prove the following:

Proposition 22. Assume that $K$ is a compact subset of $[0,1]^{\Gamma}$. Then there exists a compact, totally disconnected set $L \subset \operatorname{ad}(K)^{\mathbb{N}}$ and a continuous onto map $\phi: L \rightarrow K$ which admits a regular averaging operator.

Proof. Define $\phi^{\prime}:\{0,1\}^{\Gamma \times \mathbb{N}} \rightarrow[0,1]^{\Gamma}$ by $\phi^{\prime}(x)(\gamma)=\sum_{n \in \mathbb{N}} x(\gamma, n) r_{n}$, $r_{n}=(1 / 3)(2 / 3)^{n-1}$ and $L=\left(\phi^{\prime}\right)^{-1}(K), \phi=\phi^{\prime} \mid L$. By Proposition 18, $\phi$ admits a regular averaging operator, so it suffices to prove that $L^{\prime}$ is embeddable in $\operatorname{ad}(K)^{\mathbb{N}}$. Define $h: L \rightarrow[0,1]^{\Gamma \times \mathbb{N}}$ by

$$
h(l)(\gamma, n)= \begin{cases}\phi(l)(\gamma) & \text { if } l(\gamma, n)=1 \\ 0 & \text { if } l(\gamma, n)=0 .\end{cases}
$$

It is easy to check that $h$ is continuous. To see that it is one-to-one assume $l_{1}, l_{2} \in L$ and $l_{1} \neq l_{2}$. Then there exists $\left(\gamma_{0}, n_{0}\right) \in \Gamma \times \mathbb{N}$ such that for example $l_{1}\left(\gamma_{0}, n_{0}\right)=1$ and $l_{2}\left(\gamma_{0}, n_{0}\right)=0$. Then $h\left(l_{2}\right)\left(\gamma_{0}, n_{0}\right)=0$ and $h\left(l_{1}\right)\left(\gamma_{0}, n_{0}\right)=\phi\left(l_{1}\right)\left(\gamma_{0}\right)=\sum_{n \in \mathbb{N}} l_{1}\left(\gamma_{0}, n\right) r_{n} \geq l_{1}\left(\gamma_{0}, n_{0}\right) r_{n_{0}}>0$.

Finally $h(L) \subset \operatorname{ad}(K)^{\mathbb{N}}$, since for fixed $l \in L$ and for $n \in \mathbb{N}$, if we set $A_{n}=\{\gamma \in \Gamma: l(\gamma, n)=1\}$, then it is easy to check that $h(l)=$ $\left(\phi(l)\left\lceil A_{n}\right)_{n \in \mathbb{N}}\right.$.

REMARK 23. If $K$ is compact and its topological weight is $\mathfrak{m}$, then it can be embedded in $[0,1]^{\mathfrak{m}}$. If $i$ is such an embedding, it can be easily seen that $\operatorname{ad}(i(K))^{\mathbb{N}}$ also has topological weight $\mathfrak{m}$.

We say that a class of compacta is stable under taking Ditor spaces if for each member $K$ of $\mathcal{A}$ there exists a Ditor space for $K$, also belonging in $\mathcal{A}$.

An immediate consequence of the previous proposition is: 
Corollary 24. Assume that $\mathcal{A}$ is a class of compacta stable under taking countable products and closed subspaces. Assume also that each member $K$ of $\mathcal{A}$ can be embedded into $[0,1]^{\Gamma}$ for some set $\Gamma$ by a map $i$ such that $\operatorname{ad}(i(K))$ is also in $\mathcal{A}$. Then $\mathcal{A}$ is stable under taking Ditor spaces.

In particular the classes of Eberlein, Talagrand, Gul'ko and Corson compact spaces are all stable under taking Ditor spaces.

For further information on these classes we refer to [1], [19], [13], [8].

The proof of Theorem 4 can be derived from this corollary:

Proof of Theorem 4. It is well known (see [1]) that if $S$ is a scattered Eberlein compact space, then the closed subspaces of $\mathcal{P}(S)$ are Eberlein compact.

For the converse implication, if $K$ is Eberlein compact, let $L$ be a Ditor space for $K$ which is also Eberlein compact. Assume that $\phi: L \rightarrow K$ is continuous, onto and admits a regular averaging operator. Since $L$ is totally disconnected, we may consider $L \subset\{0,1\}^{\Gamma}$, for some $\Gamma$ such that $\Gamma=\bigcup_{n \in \mathbb{N}} \Gamma_{n}$ where the set $\left\{\gamma \in \Gamma_{n}: x(\gamma) \neq 0\right\}$ is finite for all $n \in \mathbb{N}$ and $x \in L$. By Proposition 6 there exists a continuous map $u: \mathcal{P}(K) \rightarrow \mathcal{P}(L)$, such that $\phi^{*} u=\operatorname{id}_{\mathcal{P}(K)}$. Thus $u$ is an embedding. Therefore, it suffices to embed $\mathcal{P}(L)$ into $\mathcal{P}(S)$ for some $S$ Eberlein compact and scattered.

For $A \subset \Gamma$ we denote by $\pi_{A}:\{0,1\}^{\Gamma} \rightarrow\{0,1\}^{A}$ the natural projection onto the $A$ coordinates. Let $n \in \mathbb{N}$ and set $S_{n}=\pi_{\Gamma_{n}}(L)$. Since the set $\left\{\gamma \in \Gamma_{n}: x(\gamma) \neq 0\right\}$ is finite for any $x \in L$, it easily follows that every $S_{n}$ is scattered and Eberlein compact. Identifying $\{0,1\}^{\Gamma}$ with $\prod_{n \in \mathbb{N}}\{0,1\}^{\Gamma_{n}}$, we may consider that $L \subset \prod_{n \in \mathbb{N}} S_{n}$. Next, set $L_{n}=\prod_{k=1}^{n} S_{n}$, and let $S$ be the one-point compactification of the disjoint union of $L_{n}, n \in \mathbb{N}$. Since every $L_{n}$ is a scattered Eberlein compact space, it easily follows that the same holds true for $S$.

Define now $h: C(S) \rightarrow C\left(\prod_{n \in \mathbb{N}} S_{n}\right)$ by

$$
h(f)\left(\left(s_{n}\right)_{n \in \mathbb{N}}\right)=\sum_{n=1}^{\infty} \frac{1}{2^{n}} f\left(\left(s_{k}\right)_{k=1}^{n}\right) .
$$

It is easy to verify that $h(f)$ is a continuous function on $\prod_{n \in \mathbb{N}} S_{n}$. Moreover, $h$ is positive, $\|h\| \leq 1$ and finally $h\left(1_{S}\right)=1_{\prod_{n \in \mathbb{N}} S_{n}}$. By the proof of Proposition 6, if $p \in \mathcal{P}\left(\prod_{n \in \mathbb{N}} S_{n}\right)$, then $h^{*}(p) \in \mathcal{P}(S)$. Since $h^{*}$ is weak*to-weak ${ }^{*}$ continuous it suffices to show that it is also one-to-one. Therefore it suffices to show that $h(C(S))$ is norm dense in $C\left(\prod_{n \in \mathbb{N}} S_{n}\right)$. So, pick $f \in C\left(\prod_{n \in \mathbb{N}} S_{n}\right)$ and $\varepsilon>0$. There exists an $n_{0} \in \mathbb{N}$ such that if $s, s^{\prime} \in \prod_{n \in \mathbb{N}} S_{n}$ and $\pi_{\bigcup_{n=1}^{n_{0}} \Gamma_{n}}(s)=\pi_{\bigcup_{n=1}^{n_{0}} \Gamma_{n}}\left(s^{\prime}\right)$, then $\left|f(s)-f\left(s^{\prime}\right)\right|<\varepsilon$.

Fix now $s^{0}=\left(s_{n}^{0}\right) \in \prod_{n \in \mathbb{N}} S_{n}$. Clearly the map $g: L_{n_{0}} \rightarrow \prod_{n \in \mathbb{N}} S_{n}$ satisfying $\pi_{\Gamma_{k}}\left(g\left(\left(s_{k}\right)_{k=1}^{n_{0}}\right)\right)=s_{k}$ if $k \leq n_{0}$ and $\pi_{\Gamma_{k}}\left(g\left(\left(s_{k}\right)_{k=1}^{n_{0}}\right)\right)=s_{k}^{0}$ if $k>n_{0}$, 
is an embedding of $L_{n_{0}}$ into $\prod_{n \in \mathbb{N}} S_{n}$. Define $f^{\prime}: S \rightarrow \mathbb{R}$ by

$$
f^{\prime}(x)= \begin{cases}2^{n_{0}} f(g(x)) & \text { if } x \in L_{n_{0}}, \\ 0 & \text { else. }\end{cases}
$$

Assume that $s=\left(s_{n}\right)_{n \in \mathbb{N}} \in \prod_{n \in \mathbb{N}} S_{n}$. Then by the choice of $n_{0}$ and the definition of $g$,

$$
\begin{aligned}
\left|h\left(f^{\prime}\right)(s)-f(s)\right| & =\left|\sum_{n=1}^{\infty} 2^{-n} f^{\prime}\left(\left(s_{k}\right)_{k=1}^{n}\right)-f\left(\left(s_{k}\right)_{k=1}^{\infty}\right)\right| \\
& =\left|2^{-n_{0}} f^{\prime}\left(\left(s_{k}\right)_{k=1}^{n_{0}}\right)-f\left(\left(s_{k}\right)_{k=1}^{\infty}\right)\right| \\
& =\left|f\left(g\left(s_{k}\right)_{k=1}^{n_{0}}\right)-f\left(\left(s_{k}\right)_{k=1}^{\infty}\right)\right|<\varepsilon,
\end{aligned}
$$

since $\pi_{\Gamma_{k}}\left(g\left(s_{k}\right)_{k=1}^{n_{0}}\right)=s_{k}=\pi_{\Gamma_{k}}\left(\left(s_{k}\right)_{k=1}^{\infty}\right)$ for every $k \leq n_{0}$.

For a fixed infinite set $\Gamma$ (the case we are interested in is when $\Gamma$ is uncountable) and $p \geq 1$, we denote by $B_{p}$ the unit ball of $\ell_{p}(\Gamma)$, endowed with the weak topology in the case where $p>1$, and with the weak-* topology (view $\ell_{1}(\Gamma)$ as the dual of $c_{0}(\Gamma)$ ) in the case where $p=1$. In any case $B_{p}$ can be considered to be a closed subspace of $[-1,1]^{\Gamma}$ with the pointwise topology:

$$
B_{p}=\left\{x \in[-1,1]^{\Gamma}: \sum_{\gamma \in \Gamma}|x(\gamma)|^{p} \leq 1\right\} .
$$

It is easy to check that all these spaces are homeomorphic to $B_{1}$ by the map

$$
(x(\gamma))_{\gamma \in \Gamma} \mapsto\left(\operatorname{sgn}(x(\gamma)) \cdot|x(\gamma)|^{p}\right)_{\gamma \in \Gamma} .
$$

On the other hand Remark 12 asserts that the map $\phi:\{-1,0,1\}^{\mathbb{N}} \rightarrow$ $[-1,1]$ defined by

$$
\phi(x)= \begin{cases}\sum_{n \in \mathbb{N}}|x(n)| r_{n} & \text { if } x(\min \{n \in \mathbb{N}: x(n) \neq 0\})=1, \\ -\sum_{n \in \mathbb{N}}|x(n)| r_{n} & \text { if } x(\min \{n \in \mathbb{N}: x(n) \neq 0\})=-1, \\ 0 & \text { else }\end{cases}
$$

where $r_{n}=(1-r) r^{n-1}$ for some $1 / 2<r<1$, admits a regular averaging operator. Thus by Proposition 18 the map

$$
\phi \mid \phi^{-1}\left(B_{1}\right): L=\phi^{-1}\left(B_{1}\right) \rightarrow B_{1}
$$

also admits such an operator. Observe that

$$
y \in \phi^{-1}\left(B_{1}\right) \Leftrightarrow \sum_{\gamma \in \Gamma}|\phi(y)(\gamma)| \leq 1
$$

and in any case $|\phi(y)(\gamma)|=\sum_{n \in \mathbb{N}}|y(\gamma, n)| r_{n}$. Thus

$$
L=\left\{y \in\{-1,0,1\}^{\Gamma \times \mathbb{N}}: \sum_{\gamma \in \Gamma} \sum_{n \in \mathbb{N}}|y(\gamma, n)| r_{n} \leq 1\right\}
$$


is a Ditor space for $B_{1}$ and in this case we get a very natural embedding of $L$ into the unit ball of $\ell_{1}(\Gamma \times \mathbb{N})$ which is obviously homeomorphic to $B_{1}$ :

$$
L \ni(y(\gamma, n))_{\gamma, n} \mapsto\left(y(\gamma, n) r_{n}\right)_{\gamma, n} .
$$

Unfortunately, we are not able to show that this embedding admits a regular extension operator.

It is worthwhile to observe that starting with a closed ball of radius $0<\delta<1$ in $\ell_{1}(\Gamma)$, the set

$$
L^{\prime}=\phi^{-1}\left(B_{1}(\delta)\right)=\left\{y \in L: \sum_{\gamma, n}|y(\gamma, n)| r_{n} \leq q\right\}
$$

is a Ditor space for $B_{1}(\delta)=\left\{x \in \ell_{1}(\Gamma):\|x\| \leq \delta\right\}$. This also follows from Proposition 18. Clearly, $B_{1}$ and $B_{1}(\delta)$ are homeomorphic. Hence $L^{\prime}$ may be considered as another Ditor space for $B_{1}$.

It is not clear to us what is the relation between $C(L)$ and $C\left(L^{\prime}\right)$, although $L$ and $L^{\prime}$ are Ditor spaces for the same space, both defined in a very natural way.

It remains also unclear to us if there exists any convex, non-metrizable compact subset $K$ of a Banach space and a totally disconnected space $L$ such that $C(K)$ is isomorphic to $C(L)$.

\section{References}

[1] D. Amir and J. Lindenstrauss, The structure of weakly compact sets in Banach spaces, Ann. of Math. 88 (1968), 35-46.

[2] A. Arvanitakis, A simultaneous selection theorem, to appear.

[3] Y. Benyamini, An extension theorem for separable Banach spaces, Israel J. Math. 29 (1978), 24-30.

[4] S. Ditor, On a lemma of Milutin concerning averaging operators in continuous function spaces, Trans. Amer. Math. Soc. 149 (1970), 443-452.

[5] - Averaging operators in $C(S)$ and lower semicontinuous sections of continuous maps, ibid. 175 (1973), 195-208.

[6] S. Ditor and R. Haydon, On absolute retracts, $P(S)$, and complemented subspaces of $C\left(\boldsymbol{D}^{\omega_{1}}\right)$, Studia Math. 56 (1976), 243-251.

[7] A. Etcheberry, Isomorphism of spaces of bounded continuous functions, Studia Math. 53 (1975), 103-127.

[8] M. J. Fabian, Gâteaux Differentiability of Convex Functions and Topology, WileyInterscience, 1997.

[9] R. Haydon, On a problem of Petczyński: Milutin spaces, Dugundji spaces and AE(0-dim), Studia Math. 52 (1974), 23-31.

[10] - Embedding $\boldsymbol{D}^{\tau}$ in Dugundji spaces, with an application to linear topological classification of spaces of continuous functions, ibid. 56 (1976), 229-242.

[11] C. Kuratowski, Topology, Vol. II, Academic Press, New York, 1968.

[12] J. Lindenstrauss and L. Tzafriri, Classical Banach Spaces I, II, Springer, 1977, 1979 . 
[13] S. Merkourakis, On weakly countably determined Banach spaces, Trans. Amer. Math. Soc. 300 (1987), 307-327.

[14] E. Michael, Continuous selections I, Ann. of Math. 63 (1956), 361-382.

[15] A. A. Milyutin, On spaces of continuous functions, PhD thesis, Moscow State Univ., 1952 (in Russian).

[16] A. Pełczyński, Linear extensions, linear averagings, and their applications to linear topological classification of spaces of continuous functions, Dissertationes Math. (Rozprawy Mat.) 58 (1968).

[17] D. Repovš and P. V. Semenov, Milyutin mappings and their applications, Topology Proc. 22 (1997) (summer), 385-401.

[18] D. Repovš, P. V. Semenov and E. Ščepin, On zero-dimensional Milutin maps and Michael selection theorems, Topology Appl. 54 (1993), 77-83.

[19] H. P. Rosenthal, The heredity problem for weakly compactly generated Banach spaces, Compositio Math. 28 (1974), 83-111.

[20] V. Valov, Milutin mappings and AE(0)-spaces, C. R. Acad. Bulg. Sci. 40 (1987), 9-11.

Department of Mathematics

National Technical University of Athens

15780 Athens, Greece

E-mail: sargyros@math.ntua.gr
Department of Mathematics University of Athens Panepistimiopolis 15784 Athens, Greece E-mail: aarvan@cc.uoa.gr

Received October 25, 2000

Revised version March 19, 2002 